\title{
Guidelines for the Prevention of Coronavirus Pneumonia in Elderly People
}

\author{
Jun Zhao ${ }^{a}$, Gang Xu', Congrui Feng ${ }^{a}$, Yuluo Chen ${ }^{a}$, Feng Liu ${ }^{a}$, Wei Ma ${ }^{a}{ }^{*}$ \\ ${ }^{a}$ Department of Geriatrics, National clinical key specialty, Guangzhou First People's Hospital, School of Medicine, South \\ China University of Technology; Guangzhou First People's Hospital, Guangzhou Medical University, Guangzhou 510180, \\ China.
}

\begin{abstract}
Elderly people have weakened immune functions and suffer from many underlying chronic diseases. They are at high risk of contracting COVID-19. Most of the patients who died of COVID-19 were either elderly persons or patients with underlying diseases. Therefore, it is important to improve the knowledge and awareness of the prevention of COVID-19 among the elderly. In this guideline, we analyze the susceptibility of the elderly to critical illness, and the causes of this vulnerability. Based on the characteristics of the prevention and control of COVID-19 among elderly people, we propose recommendations from three perspectives: infection source, transmission route, and susceptible population. These measures can help reduce the infection of the elderly with COVID-19.
\end{abstract}

Keywords: Elderly people, COVID-19, prevention, SARS-CoV-2

\section{Introduction}

Severe acute respiratory syndrome (SARS) coronavirus 2 (SARS-CoV-2) is a positive-sense single-stranded virus with viral envelopes covering its outer layer. It was named coronavirus owing to its petal- or club-shaped surface projections that are arranged radially on the viral envelopes, making the virus resemble a crown. The pathogens of the SARS outbreak in 2003 and the Middle East respiratory syndrome (MERS) outbreak in 2012 were both coronaviruses. Coronavirus pneumonia is a viral pneumonia associated with COVID-19, a coronavirus strain that has never been found in the human body before. Its source, characteristics, host, transmission route, and pathogenic toxicity factor, among others, are still not fully understood. The development of science and technology in fields such

\footnotetext{
* Corresponding author: Wei Ma

Mailing address: Department of Geriatrics, Guangzhou First People's Hospital, School of Medicine, South China University of Technology; Guangzhou First People's Hospital, Guangzhou Medical University, Guangzhou 510180, China.

E-mail: eymawei@scut.edu.cn

Received: 02 June 2020 / Accepted: 08 June 2020
}

as medicine has promoted a continuous improvement in global living standards and more comprehensive social welfare systems in individual countries, which have in turn prolonged the average life expectancy of human beings. Together with declines in birth and death rates, these factors have helped gradually increase the proportion of the population that is living considerably longer. China is an aging society. In the country, people over the age of 60 years are defined as elderly. According to data from the National Bureau of Statistics, as of January 2019, there were 240 million people in China who were 60 years and older, and they accounted for $17.9 \%$ of the total population. From a clinical perspective, elderly patients often differ in characteristics from young and middle-aged patients. This difference is now drawing growing attention. Elderly patients have compromised immune functions and often suffer from underlying chronic conditions, thereby becoming susceptible to infectious diseases. While the overall population is generally susceptible to COVID-19, the disease leaves elderly patients in a more critical condition after infection. Most of the patients who died of COVID-19 were either elderly persons or patients with underlying diseases. Therefore, it is important to improve the knowledge and awareness of the prevention of COVID-19 among the elderly.

A summary of the current understanding of COVID-19 is listed in Table 1 [1]. 


\section{Reasons for the susceptibility of the elderly to infection and critical illness}

The National Health Commission reported that, as of 12:00 a.m. on January 22, 2020, 425 patients had been diagnosed with COVID-19; of these individuals, 162 $(38.1 \%)$ were elderly patients aged over 65 years [2]. In addition, the average age of the first 17 patients who died of COVID-19 was 75 years (48-89 years). Among these 17 patients, 15 were aged over 60 years, and the youngest was 48 years old. Compared with patients aged under 70 years, those aged 70 years or above often have a shorter survival period (11.5 vs. 20 days). Additionally, most current deaths are associated with underlying diseases such as hypertension and diabetes [3]. These results indicate that middle-aged and elderly patients are the most susceptible to COVID-19. In addition, patients who become critically ill are mainly elderly patients with underlying diseases.

The physiological functions of elderly people degenerate as they age. For example, respiratory functions diminish, and functions of the immune system decline substantially. This functional degeneration is manifested in a decreased number of immune cells and weakened activities, making elderly people a susceptible population to infectious diseases. In addition, some elderly people suffer from various underlying diseases, such as diabetes, coronary heart disease, hypertension, and chronic lung disease. Therefore, the elderly population is more susceptible to COVID-19 and can become severely or critically ill during infection. COVID-19 has a concealed onset, a long incubation period, and diverse and atypical initial symptoms. Its primary symptom is fever that can be coupled with a mild dry cough, fatigue, breathlessness, diarrhea, etc.; other symptoms, including runny nose and sputum, are not as common. However, some COVID-19 patients may not experience a fever or cough but instead present clinical symptoms such as fatigue, chest tightness, and gastrointestinal symptoms. Symptoms in elderly people can be atypical. Some elderly patients may develop delirium and conscious disturbance during the onset or the early stage of the disease. These atypical initial symptoms made early detection, diagnosis, and intervention difficult among the first patients, thereby elevating the mortality rate of the age group. Furthermore, today, when the Internet is the main source of information, the elderly population may not always acquire timely and sufficient information compared with young and middle-aged populations. Therefore, they often lack awareness about the prevention of, and protection against, COVID-19; this increases their risks of infection and disease transmission. Consequently, elderly people's knowledge and awareness of the prevention of COVID-19 should be strengthened.

\section{Guidelines for the prevention of COVID-19 among elderly people}

Table 1. Clinical characteristics of COVID-19.

\begin{tabular}{|c|c|}
\hline Project & Description \\
\hline Pathogenic characteristics & $\begin{array}{l}\text { - } \beta \text {-coronavirus. } \\
\text { - Host remains unidentified. Existing research indicates that its homology with SARS-like coronavirus in bats is above } 85 \% \text {. } \\
\text { - Sensitive to ultraviolet light and heat. It can be inactivated by heating at } 56^{\circ} \mathrm{C} \text { for } 30 \text { minutes, ether, } 75 \% \text { ethanol, chlorine- } \\
\text { containing disinfectants, peracetic acid, and lipid solvents (such as chloroform). Chlorhexidine cannot inactivate the virus. }\end{array}$ \\
\hline Infection source & Patients infected with SARS-CoV-2, although asymptomatic virus carriers, can also be the infection source. \\
\hline Transmission route & $\begin{array}{l}\text { Primary transmission routes include respiratory droplet transmission and contact transmission. The possibility of aerosol } \\
\text { and digestive tract transmission is yet to be confirmed. }\end{array}$ \\
\hline Susceptible population & General population. \\
\hline Incubation period & Generally, 1-14 days. Most patients develop symptoms within 3-7 days. \\
\hline Clinical manifestations & $\begin{array}{l}\text { - Fever, fatigue, and dry cough are major manifestations. Some patients may also develop symptoms such as nasal } \\
\text { congestion, runny nose, sore throat, and diarrhea. } \\
\text { - Severe cases often develop dyspnea and/or hypoxemia one week after disease onset. In critical cases, the disease may } \\
\text { rapidly progress to acute respiratory distress syndrome, septic shock, coagulopathy, and metabolic acidosis that is difficult } \\
\text { to treat. } \\
\text { - Mild cases demonstrate symptoms such as low fever and minor fatigue but no pneumonia symptoms. } \\
\text { - Most patients have a good prognosis, and children often demonstrate relatively mild symptoms. Few patients will progress } \\
\text { to critical conditions. However, elderly patients and those with underlying chronic diseases typically have a poor prognosis. }\end{array}$ \\
\hline Laboratory examinations & $\begin{array}{l}\text { - During the early stage of disease onset, the total number of peripheral white blood cells is normal or reduced, and the } \\
\text { lymphocyte count decreases. Some patients may have increased levels of liver enzymes, lactate dehydrogenase (LDH), } \\
\text { muscle enzymes, and myoglobin. Some critically ill patients can also experience increased levels of troponin. } \\
\text { - In most patients, C-reactive protein (CRP) levels and erythrocyte sedimentation rates increase, and procalcitonin stays } \\
\text { normal. In critical cases, D-dimer levels increase, and the number of peripheral blood lymphocytes progressively declines. } \\
\text { - The nucleic acid of SARS-CoV-2 can be detected in nasal and pharyngeal swabs, sputum, lower respiratory tract } \\
\text { secretions, blood, feces, etc. }\end{array}$ \\
\hline Imaging & $\begin{array}{l}\text { During early onset, images show multiple small, patchy shadows and interstitial changes that are highly evident in the } \\
\text { peripheral lung. As the disease progresses, images show multiple ground-glass and infiltration shadows in both lungs. In } \\
\text { critical cases, lung consolidation can be observed, although pleural effusion is rare. }\end{array}$ \\
\hline Treatment & As there are currently no specific antiviral drugs, symptomatic treatment is the primary treatment method. \\
\hline
\end{tabular}


Table 2. Guidelines for the prevention of COVID-19 among different age groups.

\begin{tabular}{lll}
\hline & Young and middle-aged population & Elderly population \\
\hline Knowledge of disease prevention & Obtains frequently updated knowledge from Often lacks knowledge and awareness of the prevention \\
& multiple sources & of COVID-19 due to insufficient knowledge source \\
Prevention initiatives & Flexible and active & Often relies on others \\
Immunity & Normal & Decreased \\
Combined underlying chronic diseases & Less & More \\
Clinical manifestations & More typical, easy to detect & Less typical and more concealed, easy to ignore \\
Critical cases & Less common & More common \\
\hline
\end{tabular}

Based on the characteristics of the prevention and control of COVID-19 among elderly people (Table 2), we propose the following recommendations from three perspectives: infection source, transmission route, and susceptible population.

\section{Prevention of contact with the infection source}

Multiple animal species, including humans, can be infected with SARS-CoV-2. Although the natural host of the virus has still not been identified, some evidence suggests that the Chinese rufous horseshoe bat (Rhinolophus sinicus) may be the natural host of the virus, whilst other wild animals may also participate in the transmission. Therefore, the following precautions are advised. People should wash hands after touching poultry and livestock, avoid direct contact with sick animals and poultry, not eat wild animals, not eat raw or undercooked poultry and livestock meat, not process and eat dead poultry and livestock (or poultry and livestock meat that has not been quarantined). Since elderly people are often assisted by caregivers or helpers with their diets and activities of daily life, they should also be educated about the prevention of $\mathrm{CO}$ VID-19.

COVID-19 patients and carriers are the primary spreaders of the disease. Therefore, curing or isolating them is an effective method of stopping the transmission of $\mathrm{CO}$ VID-19. Due to their weakened body response, elderly people often demonstrate an underlying onset and atypical clinical symptoms. Consequently, it is necessary to closely monitor elderly people with histories of disease exposure, and comprehensive screening should be performed if necessary. As the incubation period of COVID-19 can be as long as 14 days, potential carriers should be closely monitored for 14 days to identify virus carriers.

\section{Breaking of the transmission route}

SARS-CoV-2 is highly pathogenic and contagious. Its transmission routes include airborne droplet, contact, and potential fecal-oral transmission.

As the primary transmission route of SARS-CoV-2, airborne droplet transmission can happen during face-to-face conversations, sneezing, and coughing in daily life. The most effective method of reducing airborne droplet transmission is to wear masks correctly. In addition, people should avoid spitting in public spaces. Covering the nose and mouth while sneezing can also effectively decrease the chance of droplet transmission. Due to their reduced ability to excrete sputum, elderly people sometimes need assistance in doing so. During this process, procedures for auxiliary sputum discharge should be strictly followed to protect both the operator and the patient and reduce the risk of infection. SARS-CoV-2 can survive in air for short periods and form particles that can spread through the air and infect other people through long-distance transmission. Therefore, keeping the environment ventilated, avoiding crowded places, and adhering to self-isolation are effective ways of reducing the chance of airborne transmission. During ventilation, excessive temperature changes should be avoided.

SARS-CoV-2 can also spread through contact transmission via skin and conjunctiva. To reduce contact transmission, individuals should wash hands frequently, maintain good hand hygiene, regularly perform indoor cleaning, keep the environment clean and tidy, reduce indoor environmental pollution, and promptly remove household garbage and waste. In addition, disinfecting frequently touched items with alcohol can effectively inactivate the virus.

Recent tests suggest that SARS-CoV-2 can be isolated in patients' feces. As the possibility of fecal-oral transmission cannot be eliminated, it is important to maintain good personal hygiene and prevent the disease from entering the body via the mouth. After using the toilet, people should cover the bowl with the lid before flushing to prevent the aerosol from spreading into the air.

Elderly people are physically weak and have poor adaptability to the environment. In particular, as they cannot adapt to environmental temperature changes, they are susceptible to cold-induced respiratory infectious diseases. In addition, due to their poor self-care ability, elderly people inevitably need caregivers and therefore cannot absolutely self-isolate. Therefore, the health of caregivers should also be ensured to cut off the transmission route of COVID-19 among the elderly.

\section{Protection of the susceptible population}

Based on the characteristics of elderly people, the following recommendations are proposed to reduce the incidence of COVID-19.

\section{(1) Reasonable diet}

Malnutrition is a main factor affecting disease outcome in elderly patients [4]. Therefore, elderly people should maintain a balanced diet; control their body mass index 
(BMI) within the $18.5-24 \mathrm{~kg} / \mathrm{m} 2$ range; have a balanced intake of calories, protein, vitamins, minerals, etc.; and have more meals a day but less food at each for example, eat five smaller meals. In addition, they need to eat food that is easy to digest or food that facilitates digestion; increase their consumption of vegetables and fruits; drink water frequently; and consume high-protein foods, such as fish, meat, eggs, milk, beans, and nuts, in reasonable quantities on a daily basis. They should strictly avoid eating wild animals, rotten and expired food, and semi-cooked or raw food. It is important to ensure rich and diverse food types and sources and maintain a balanced diet composed of both meat and vegetables so that adequate nutrition is supplied to the body. For elderly people with dysphagia, nasal feeding can be performed according to their dieticians' recommendation, whilst parenteral nutrition support can be administered if necessary. For all elderly people, accidental inhalation during eating should be prevented because it can cause aspiration pneumonia.

\section{(2) Good living habits}

Elderly people need adequate sleep every day and warmth during sleep. In addition, it is important to select an appropriate method of exercising, , exercise moderately, and maintain a healthy lifestyle, thereby improving resistance to infection. The elderly should also avoid going outside, especially crowded places, such as senior activity centers and senior universities. They should frequently open windows to keep their rooms well ventilated, disinfect their rooms regularly, avoid spitting in areas frequented by others, maintain good hand hygiene, actively monitor their personal health, and measure their body temperature if experiencing a fever. Elderly people who are bedridden and have limited physical mobility should undergo limb rehabilitation, and their bodies should be turned regularly in bed to prevent deep vein thrombosis and bedsores.

\section{(3) Healthy mental state}

Psychological crisis intervention should be incorporated into the deployment of epidemic prevention and control to reduce the possible psychological trauma that can result from the disease [5]. After retirement, the activity range of elderly people decreases, and their focus on activity changes. Together with changes in their physiological characteristics, such as sensation, exercise, and cognition, elderly people experience changes in psychological characteristics, which are demonstrated by reduced security and adaptability. As a result, they tend to develop feelings of loss, inferiority, emptiness, etc. Upon disease onset, elderly people can exhibit higher levels of anxiety and depression and have difficulty in cooperating with treatment. Therefore, it is important to provide the elderly population with targeted medical and entertainment services, provide them regular care in their living environment, encourage them to demonstrate their talents and hobbies, and persuade them to actively adapt to a new lifestyle. By implementing these methods, elderly people can maintain contact with their families and society, and their anxiety and depression can be alleviated so that they can maintain a healthy mental state and actively cooperate with their treatment.

\section{(4) Active treatment of underlying diseases}

Elderly patients often suffer from underlying chronic diseases, such as hypertension, diabetes, and coronary heart disease, making them susceptible to infection and critical illness during the COVID-19 pandemic. Therefore, these patients should follow doctors' instructions and administer drugs promptly, regularly, and properly based on the specific underlying disease. Secondary prevention and treatment of related diseases should be carefully administered. In addition, these patients should learn to evaluate their symptoms, monitor their general condition, and promptly seek medical advice when there is a change in their condition. They should appropriately describe their condition during consultations and avoid consulting incorrect doctors or concealing their sickness. For elderly patients with limited activity and impaired cognition, family members should assist with treatment and properly evaluate and monitor the patient.

\section{Discussion}

With increasing social aging, the health problems of elderly people are receiving increasing attention. Elderly people have poor resistance and often suffer from other underlying diseases. Therefore, during the COVID-19 outbreak, the elderly population presents general susceptibility, high prevalence, fast disease progression, and high mortality. This makes them a key target for epidemic prevention. Unlike younger adults, elderly people have reduced body function, declined infection resistance, and limited self-care ability. Therefore, based on guidelines for the prevention of COVID-19 among adults [1], we propose prevention recommendations tailored for the elderly population in this study. The proposed recommendations ensure that epidemic prevention measures protect not only elderly patients but also their caregivers. They also guarantee that comprehensive, precise, and focused care is provided to these patients to eradicate COVID-19 as early as possible.

\section{Declarations}

Conflict of interest: All authors declared that there are no conflicts of interest.

Authors' contributions: Made substantial contributions to conception and design of the study: Feng Liu, Wei Ma; Performed material acquisition, as well as provided technical and information support: Jun Zhao, Gang Xu, Congrui Feng, Yuluo Chen.

Financial support and sponsorship: This work was supported by Guangdong Planned Project of Science and 
Technology (No. 2017A020215007), Guangzhou General Science and Technology Project of Health and Family Planning (No. 20171A011246), and Guangdong Science and Research Project of Traditional Chinese Medicine Bureau (No. 20182104).

\section{References}

1. General Office of the National Health Commission, General Office of the National Administration of Traditional Chinese Medicine. Diagnosis and Treatment Guidelines for COVID-19 (5th Trial Edition) [EB/OL] (2020-02-03).

2. Li Q, Guan $\mathrm{X}, \mathrm{Wu} \mathrm{P}$, et al. Early transmission dynamics in Wuhan, China, of novel coronavirus-infected pneumo- nia. New England Journal of Medicine, 2020.

3. Wang W, Tang J, Wei F. Updated understanding of the outbreak of 2019 novel coronavirus (2019-nCoV) in Wuhan, China. Journal of medical virology, 2020, 92(4): 441-447.

4. Geriatric Nutrition Support Group, Parenteral and Enteral Nutrition Branch of the Chinese Medical Association. Chinese Expert Consensus on Parenteral and Enteral Nutrition Support for Elderly Patients. Chinese Journal of Geriatrics, 2013, 32(9): 913-929.

5. National Health Commission. Guiding Principles for Psychological Crisis Interventions during COVID-19 Outbreak [EB/OL]. http://www.nhc.gov.cn/jkj/s3577/2 02001/6adc08b966594253b2b791be5c3b9467.shtml. (2020-01-27).

Cite this article as: Zhao $\mathrm{J}, \mathrm{Xu} \mathrm{G}$, Feng $\mathrm{C}$, et al. Guidelines for the Prevention of Coronavirus Pneumonia in Elderly People[J]. Aging Pathobiology and Therapeutics, 2020, 2(2): 73-77. 